\title{
A CMOS Quadrature Signal Generator for Impedance Spectroscopy Measurement Systems
}

\author{
Alejandro Márquez, Nicolás Medrano, Belén Calvo, Pedro A. Martínez \\ Grupo de Diseño Electrónico (GDE) \\ Instituto de Investigación en Ingeniería de Aragón (I3A) \\ Universidad de Zaragoza, Mariano Esquillor s/n, 50018, Zaragoza, Spain. \\ Tel.+34-976762707, e-mail: amarquez@unizar.es
}

\begin{abstract}
A CMOS fully integrated quadrature signal generator for on-chip impedance spectroscopy (IS) applications is presented. Frequency can be digitally tuned from 5 to $350 \mathrm{kHz}$ with 12-bit resolution. Power consumption is $0.77 \mathrm{~mW}$ and active area is $0.129 \mathrm{~mm}^{2}$. Its suitability for the target application is validated with a Randles test impedance cell modelling a protein.
\end{abstract}

\section{Motivation and design}

Recent advances in microsensing techniques are leading to a growing need for on-chip electronic instrumentation, not only providing the required performances but simultaneously complying with the constraints of low power and compact size, to fully satisfy the emerging market demands and potential applications of portable and wearable sensing devices. New transduction techniques in micro-integrated sensors include resonant detection and complex impedance characterization. Thus, a suitable interrogation approach, which presents advantages compared to other electronic readout techniques due to its characteristics, is synchronous demodulation. This technique can be implemented in CMOS technology [1] using phase sensitive detection (PSD) or quadrature modulators, which extract the signal amplitude and phase information at a specific frequency $f_{o}$ while noise signals at other frequencies are rejected. Figure 1 illustrates the conceptual scheme of a dual PSD module. A device sensor is excited by an input signal $V_{S}$, providing an output $V_{Z}$ that is next multiplied by two 90 degrees shifted reference signals also with frequency $f_{o}$. The resulting mixer outputs are finally low-pass filtered, obtaining two DC values $V_{X}$ and $V_{Y}$, proportional to the processed signal amplitude and phase, so that the magnitude and phase, or equivalently, the real and imaginary part of the sensor impedance can be obtained.

Accordingly, in dual PSD-based conditioning electronics, the actuation system requires the generation of two 90 degrees shifted signals $V_{S}$ and $V_{C}$, so that one of them is also used as the sensor excitation. Most of microelectronic implementations of synchronous demodulators integrate the read-out circuit together with the transducer in the same chip, but leave the actuation system out of the integrated circuit, using external signal generators, thus increasing size, power consumption and complexity.

Therefore, a fully integrated PSD instrument will require the design of a suitable integrated actuation system. This work presents the implementation and experimental results of a self-contained versatile CMOS signal generation circuit for portable PSD applications. It is based on a digitally programmable analog quadrature sinusoidal oscillator (Figure 2), based on a single supply active-RC two integrator loop using three operational amplifiers (OpAmps) as active cells, two capacitors and six resistances: resistors $R_{A}, R_{B}$ and $R_{1}$ are passive, while $R_{E Q}$ are identical active resistors, respectively conformed by a passive resistor $R$ in series with a digital control cell using a current summing/division network (CS/DNs) [2]. It generates two sinusoidal signals in quadrature (Figure 3), whose frequency is digitally controlled by a 12-bit custom digital-to-analog (DAC) architecture based on CS/DNs over a wide range from 10 to $350 \mathrm{kHz}$, covering most typical impedance sensor operation. It has been fabricated in a $1.8 \mathrm{~V}-0.18 \mu \mathrm{m}$ CMOS technology, featuring low-power $(0.77 \mathrm{~mW})$ and compact size $\left(0.129 \mathrm{~mm}^{2}\right)$, to be suitable for applications in portable on-chip systems.

\section{Impedance measurements}

The suitability of the proposed quadrature oscillator as signal source for an IS micro-instrument is herein analyzed. The selected test impedance configuration emulates the real impedance configuration known as Randles cell modelling a protein [3]: the target impedance $Z$ (Figure 4) is connected to a standard setup circuit consisting on an OpAmp, configured as an auto-balancing bridge with a feedback resistor $R_{F}=429 \mathrm{k} \Omega$. Protein model values are: $R_{S}=$ $149 \mathrm{k} \Omega, R_{P}=431 \mathrm{k} \Omega$ and $C_{P}=57 \mathrm{pF}$.

It is possible to recover both magnitude and phase by applying a synchronous quadrature demodulator using the signals provided by the proposed actuation circuit. Besides, the feasibility of applying this 
technique for impedance characterization is verified using a commercial LCR-Meter. Experimental results prove that both module and phase are successfully recovered with relative errors below 5\% (Figure 5). Global characteristics make this design a highly suitable choice as signal generation module for instrument-on-a-chip devices. To the best authors' knowledge, this is the unique selfcontained quadrature actuation system, low-voltage low-power compatible and featuring such high frequency resolution over such a wide linear tuning range.

\section{Conclusions}

A $1.8 \mathrm{~V}-0.18 \mu \mathrm{m}$ CMOS digitally programmable quadrature oscillator has been designed, fabricated and tested to be used as self-contained actuation system for on-chip IS applications over a wide frequency range with low-power and low-area consumption. Experimental tests show good performance as self-contained signal generator in general purpose impedance PSD-based measurement applications, as proved the comparison with the results achieved using a commercial LCR-meter.

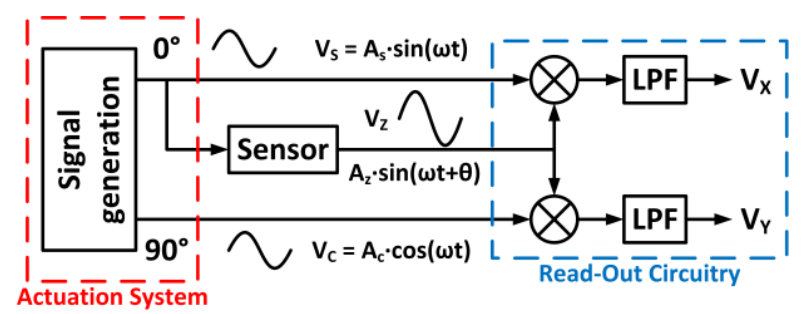

Figure 1. Scheme of the dual phase sensitive (PSD) detection.

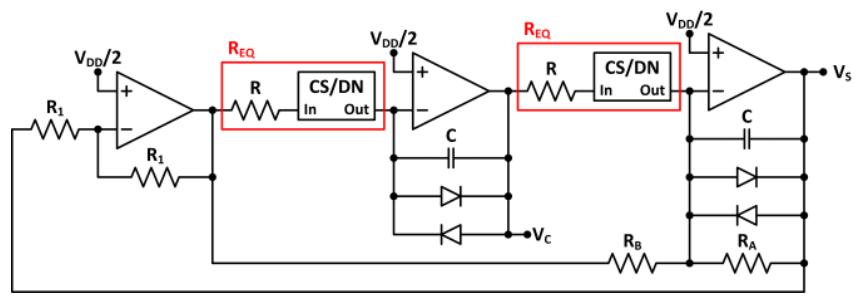

Figure 2. Programamble quadrature oscillator topology.

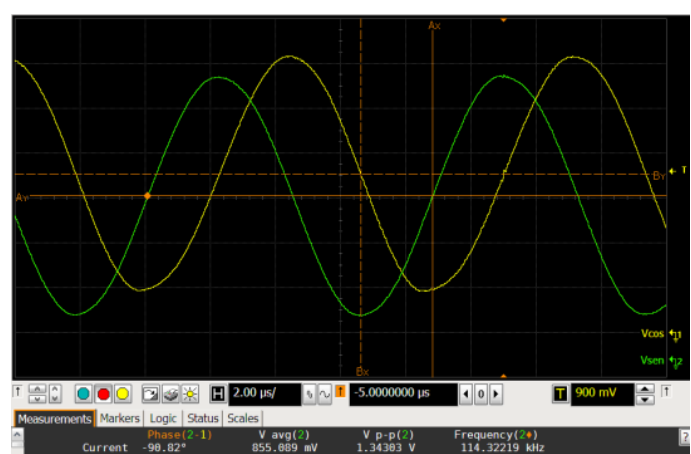

Figure 3. Output quadrature signals for digital code \#500. Oscillation frequency is $114.3 \mathrm{kHz}$ and phase shift is $-90.82^{\circ}$.

\section{REFERENCES}

[1]. D. GARCÍA-ROMEO, M. R. VALERO, N MEDRANO, B. CALVO, and S. CELMA. A High Performance LIA-Based Interface for Battery Powered Sensing Devices. Sensors, vol. 15, no. 10, pp. 25260-25276, 2015. DOI: 10.3390/s151025260.

[2]. A. MÁRQUEZ, J. PÉREZ-BAILÓN, P. A. MARTÍNEZ, B. CALVO, and N. MEDRANO. A CMOS low-power 12-bit digitally programable analog sinusoidal actaution system. IEEE International Instrumentation and Measurement Technology Conference, 2017. DOI: 10.1109/I2MTC.2017.7969836.

[3]. A. MANICKAM, A. CHEVALIER, M MCDERMOTT, A. D. ELLINGTON, and A. HASSIBI. A CMOS Electrochemical Impedance Spectroscopy (EIS) Biosensor Array. IEEE Transactions on Biomedical Circuits and Systems, vol. 4, no. 6, pp. 379-390, 2010. DOI: 10.1109/TBCAS.2010.2081699.

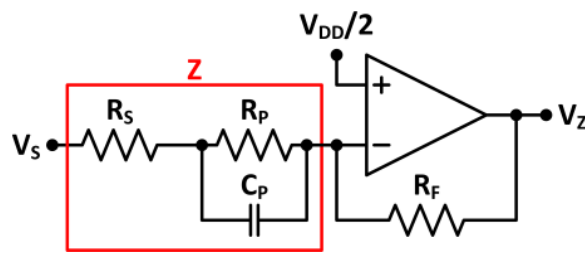

Figure 4. Test impedance $Z$ Randles cell.

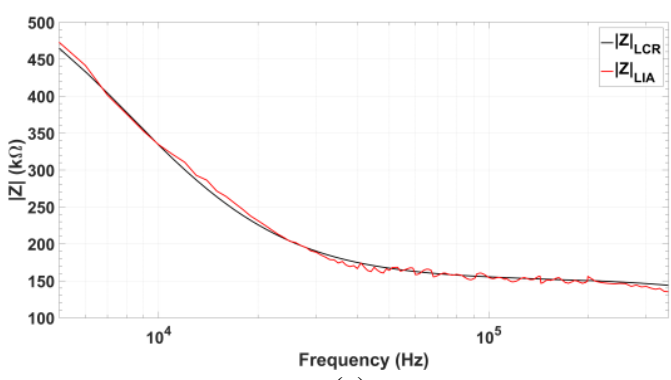

(a)

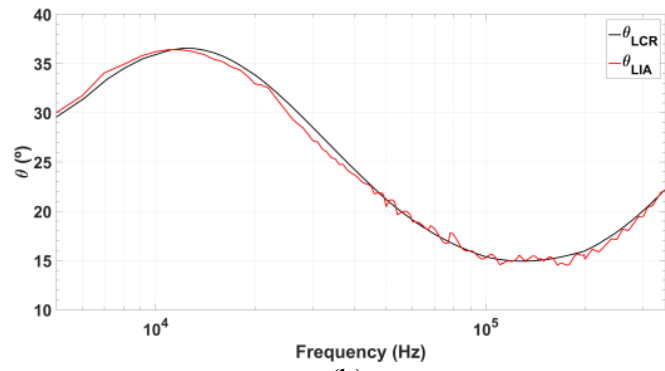

(b)

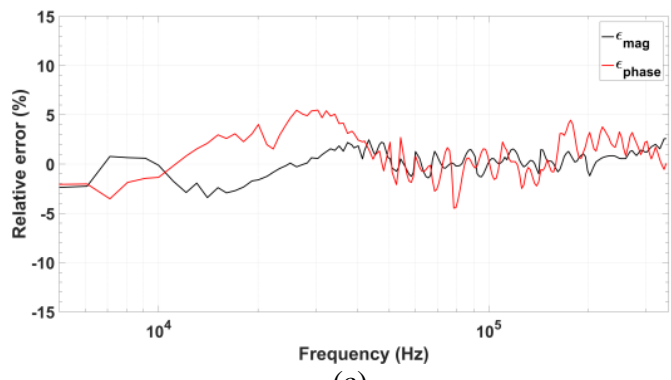

(c)

Figure 5. Impedance measurement using the proposed QO (red), compared to a commercial LCR-meter: a) magnitude, b) phase, and c) relative errors. 